\title{
On the decay of inhomogeneous turbulence
}

\author{
By J. R. CHASNOV \\ Department of Mathematics, The Hong Kong University of Science and Technology, \\ Clear Water Bay, Kowloon, Hong Kong
}

(Received 7 January 1997)

The decay of high-Reynolds-number inhomogeneous turbulence in an unbounded domain is considered. The turbulence may be initially localized in one to three spatial directions and the fluid is assumed to be at rest at infinity in those directions. Previous arguments used to determine the decay laws of homogeneous turbulence are extended to the decay of inhomogeneous turbulence by integrating the turbulence statistics over the inhomogeneous directions. Dimensional arguments based on the invariance or near-invariance of low-wavenumber spectral coefficients associated with the integrated mean-square velocity are used to determine asymptotic decay laws for inhomogeneous turbulence. These decay laws depend on the number of inhomogeneous directions of the flow field and reduce to the well-known decay laws of homogeneous turbulence when this number is zero. Different decay laws are determined depending on the spectral behaviour at low wavenumbers. Asymptotic similarity states of the spectrum during the decay and of the distribution of the mean-square velocity along the inhomogeneous directions are also determined. An analytical result for the decay of the mean-square velocity at the centre of the initial disturbance is found, and the decay proceeds more rapidly with increasing number of inhomogeneous directions due to the transport of energy along those directions.

Large-eddy simulations of decaying turbulence homogeneous in a plane and localized in a single direction are performed to test the theoretical scaling laws. The numerically determined asymptotic decay laws of the integrated mean-square velocity agree well with the theoretical predictions. A self-similar decay of the spectra and mean-square velocity distributions is also observed. The simulation results suggest that when the low-wavenumber spectral coefficient is an exact invariant, a unique similarity state depending only on the initial value of this invariant and independent of all other aspects of the initial conditions is attained asymptotically.

\section{Introduction}

The simplest type of inhomogeneous turbulence occurs in an unbounded fluid in which an initial random disturbance to the fluid velocity is localized in one or more spatial directions. The mean-square velocity of the fluid at a fixed point then decays as a consequence of both viscous dissipation and the outward transport of energy from turbulent to less turbulent regions along the directions of statistical inhomogeneity. Analytical solutions for the final period of decay of this simplest type of inhomogeneous turbulence were obtained by Phillips (1956), and the main purpose of this work is to extend his low-Reynolds-number final period results to high Reynolds numbers. 
Decaying inhomogeneous turbulence in an unbounded domain is a close relative of decaying homogeneous turbulence, for which the rate of decay of the mean-square velocity is the same at all points in the fluid. High-Reynolds-number decay laws of the mean-square velocity in homogeneous turbulence are well-known (Kolmogorov 1941; Saffman 1967b), and large-eddy simulations have verified those laws to within a few percent (Chasnov 1994). The fundamental difference between homogeneous turbulence and the inhomogeneous turbulence considered here is the spatial transport of energy from active turbulent regions to quiescent fluid. That transport may be along a single axis if the turbulence is initially homogeneous in a plane, or may be in all three directions if the initial turbulent motion is completely localized.

Our problem with a single inhomogeneous direction shares common features with the shearless turbulence mixing layer, for which experimental and numerical data exist (Gibert 1980; Veeravalli \& Warhaft 1989; Briggs et al. 1996). In both problems the turbulence is inhomogeneous along a single direction and there is an absence of boundaries and mean shear. The essential difference is the behaviour of the fluid at infinity along the inhomogeneous direction. In the mixing layer the fluid motion approaches homogeneous turbulence at infinity whereas in the problem studied here the fluid approaches a state of rest.

The equations governing the decay of the mean-square velocity of inhomogeneous turbulence for which the fluid is at rest at infinity along the inhomogeneous directions may be made similar to that for homogeneous turbulence if the mean-square velocity is integrated over the inhomogeneous directions of the turbulence. The extra transport terms in the equations are then converted to surface integrals which vanish. Scaling arguments previously applied to homogeneous turbulence may then be used to determine the evolution of the integrated mean-square velocity. We will show that the generalized scaling laws thus obtained define different asymptotic similarity states for freely decaying turbulence with zero to three directions of inhomogeneity.

The first part of this work, presented in $\S 2$ and $\S 3$, contains a heuristic derivation of the analytical scaling laws for decaying inhomogeneous turbulence. The second part, presented in $\$ 4$ and $\S 5$, details the method and results of large-eddy simulations designed to test some of the predicted decay laws. The simulations consider an initial random flow field which is homogeneous in a plane and localized in a single direction, with a given spectrum and mean-square velocity distribution.

\section{Fundamentals and statistics}

The Navier-Stokes and continuity equations governing an incompressible fluid's motion are given by

$$
\begin{gathered}
\frac{\partial u_{i}}{\partial t}+u_{j} \frac{\partial u_{i}}{\partial x_{j}}=-\frac{1}{\rho_{0}} \frac{\partial p}{\partial x_{i}}+v \frac{\partial^{2} u_{i}}{\partial x_{j} \partial x_{j}}, \\
\frac{\partial u_{i}}{\partial x_{i}}=0,
\end{gathered}
$$

from which an evolution equation for the mean-square velocity at a fixed spatial point may be obtained:

$$
\frac{\partial}{\partial t}\left\langle\boldsymbol{u}^{2}\right\rangle+\frac{\partial}{\partial x_{j}}\left\langle u_{j} \boldsymbol{u}^{2}+\frac{2}{\rho_{0}} u_{j} p-v \frac{\partial}{\partial x_{j}} \boldsymbol{u}^{2}\right\rangle=-2 v\left\langle\left(\partial u_{i} / \partial x_{j}\right)^{2}\right\rangle
$$


where $\boldsymbol{u}^{2}=u_{i} u_{i}$, and $\langle\ldots\rangle$ denotes an ensemble average, or equivalently an average over the spatial coordinates for which the turbulence is homogeneous. Inhomogeneous turbulence in an unbounded domain differs from homogeneous turbulence due to the additional transport terms on the left-hand side of (2.3), which are non-zero only for derivatives taken along the inhomogeneous directions. Further integration of (2.3) over the $n$ inhomogeneous spatial coordinates eliminates the transport term since the fluid is at rest at infinity, and one obtains

$$
\frac{\mathrm{d}}{\mathrm{d} t} \int\left\langle\boldsymbol{u}^{2}\right\rangle \mathrm{d}^{n} x=-2 v \int\left\langle\left(\partial u_{i} / \partial x_{j}\right)^{2}\right\rangle \mathrm{d}^{n} x .
$$

We define $e_{n}$ to be one-half the integrated mean-square velocity of our inhomogeneous turbulence:

$$
e_{n}=\frac{1}{2} \int\left\langle\boldsymbol{u}^{2}\right\rangle \mathrm{d}^{n} x,
$$

and (2.4) shows that $e_{n}$ monotonically decreases in time due to viscous dissipation. The corresponding spectrum $E_{n}(k)$ of wavenumber magnitude $k$ may be defined from the spherically integrated Fourier transform of the integrated two-point velocity correlation as

$$
E_{n}(k, t)=\frac{1}{(2 \pi)^{3}} \iiint \frac{1}{2}\left\langle u_{i}(\boldsymbol{x}) u_{i}(\boldsymbol{x}+\boldsymbol{r})\right\rangle \exp (-\mathrm{i} \boldsymbol{k} \cdot \boldsymbol{r}) \mathrm{d}^{n} x \mathrm{~d} \boldsymbol{r} \mathrm{d} A(k),
$$

where the integral $\mathrm{d} A$ is over the surface of a sphere in Fourier space of radius $k$. The integral of $E_{n}(k, t)$ over $k$ yields $e_{n}$, so that (2.4) may be rewritten in terms of the spectrum $E_{n}$ as

$$
\frac{\mathrm{d}}{\mathrm{d} t} \int E_{n}(k, t) \mathrm{d} k=-2 v \int k^{2} E_{n}(k, t) \mathrm{d} k .
$$

It will be useful to define two characteristic length scales of the flow field: one an integral scale of the turbulence itself, and the other representative of the spatial inhomogeneity. The characteristic length scale of the turbulence is defined by integrating the (integrated) velocity product over a separation length $r$ and averaging over all the directions of $\boldsymbol{r}$. Averaging over all the directions is physically meaningful only if the integral scales of the turbulence in the homogeneous and inhomogeneous directions grow at the same rate, and our later numerical experiments have indicated that this is indeed the case. The integral scale of the turbulence $l(t)$ is thus defined as

$$
l=\frac{1}{2 e_{n}} \iint \frac{\left\langle u_{i}(\boldsymbol{x}) u_{i}(\boldsymbol{x}+\boldsymbol{r})\right\rangle}{4 \pi r^{2}} \mathrm{~d} x^{n} \mathrm{~d} \boldsymbol{r}
$$

and is related to the spectrum by

$$
l=\frac{\pi}{2 e_{n}} \int_{0}^{\infty} k^{-1} E_{n}(k) \mathrm{d} k .
$$

It is clear that $l$ is a characteristic length of the scale sizes which contribute most to $e_{n}$. The representative length scale of the spatial inhomogeneity is defined as

$$
d=\left[\frac{3}{8 n e_{n}} \int|\boldsymbol{x}|^{2}\left\langle\boldsymbol{u}^{2}\right\rangle(\boldsymbol{x}) \mathrm{d}^{n} x\right]^{1 / 2}
$$

where the integration is over the inhomogeneous directions with origin at the centre of the disturbance. The length scale $d$ is directly proportional to the width of the spatial inhomogeneity, and this will be shown explicitly for a particular choice of 
initial conditions in $\S 4$. Our choice of constants in (2.10) is such that $l$ and $d$ are bounded by the same maximum value in our later numerical simulations performed in triply periodic domains. More details on this latter point can also be found in $\S 4$.

\section{Scaling laws}

In previous work (Chasnov 1994), we argued that the final period of decay laws for homogeneous turbulence (Batchelor 1953) can motivate a heuristic derivation of the high-Reynolds-number decay laws using simple scaling arguments based on lowwavenumber spectral coefficients of the turbulence energy spectrum. The asymptotic final period of decay laws necessarily depends linearly on these low-wavenumber spectral invariants: the equations of motion are linear in the final period of decay, and viscous forces preferentially dissipate energy at high wavenumbers. In addition to a linear dependence on the low-wavenumber spectral coefficient, the energy decay law only depends on the kinematic viscosity $v$ of the fluid and the evolution time $t$. A similar scaling law can be advanced at high Reynolds numbers where the molecular viscosity is conceptually replaced by a turbulent viscosity and thus no longer enters the scaling as an independent parameter. The absence of the molecular viscosity from the high-Reynolds-number scaling laws is in accord with the usual turbulence phenomenology that the energy dissipation rate approaches a non-zero constant in the limit of zero viscosity. It is thus reasonable that the energy decay law at high Reynolds number now depends nonlinearly on the low-wavenumber spectral coefficients and on the time $t$ alone. Decay laws of the mean-square velocity of homogeneous turbulence previously determined by Kolmogorov (1941) and Saffman $(1967 b)$ may be rederived in this way.

In considering the final period of decay of inhomogeneous turbulence, Phillips (1956) showed that the decay laws again depend linearly on low-wavenumber invariants, and on viscosity and time $t$. During the final period for which the equations are linear, (2.7) holds at each $k$ so that

$$
\frac{\partial}{\partial t} E_{n}(k, t)=-2 v k^{2} E_{n}(k, t) .
$$

Equation (3.1) may be solved analytically provided the form of the initial spectrum upon entering the final period is known. A long-time solution only requires the form of the spectrum near $k=0$, and Phillips considered directly an expansion of the vorticity and velocity fields near zero wavenumber for a localized velocity disturbance. Here, we simply assume that an expansion of the spectrum $E_{n}(k, t)$ near $k=0$ may be given by

$$
E_{n}(k, t)=2 \pi \sigma^{n} k^{2}\left(B_{0}+B_{2} k^{2}+\ldots\right),
$$

where $\sigma$ is a length scale representative of the inhomogeneity. As in the work of Batchelor \& Proudman (1956), Phillips took $B_{0}$ to be identically zero when the turbulence was homogeneous in one or more directions, but Saffman (1967a) later demonstrated that this was not necessarily so. Saffman also showed that $B_{0}$ is strictly invariant in time and his argument still applies to flows which are inhomogeneous in one or more directions. When $B_{0}$ is initially zero, nonlinear transfer processes result in $B_{2}(t) \neq 0$. In the final period however, when the equations of motion are linear, $B_{2}$ is independent of time. The exact asymptotic final period of decay laws may now be determined analytically from (3.1) and one finds

$$
e_{n} \propto\left(\sigma^{n} B_{0}\right)(v t)^{-3 / 2} \text { or } e_{n} \propto\left(\sigma^{n} B_{2}\right)(v t)^{-5 / 2} .
$$




\begin{tabular}{ccc}
\multicolumn{3}{c}{$B_{0} \neq 0$} \\
\cline { 3 - 3 }$n$ & $e_{n} \propto$ & $l \propto$ \\
0 & $B_{0}^{2 / 5} t^{-6 / 5}$ & $B_{0}^{1 / 5} t^{2 / 5}$ \\
1 & $\left(\sigma B_{0}\right)^{1 / 2} t^{-1}$ & $\left(\sigma B_{0}\right)^{1 / 6} t^{1 / 3}$ \\
2 & $\left(\sigma^{2} B_{0}\right)^{4 / 7} t^{-6 / 7}$ & $\left(\sigma^{2} B_{0}\right)^{1 / 7} t^{2 / 7}$ \\
3 & $\left(\sigma^{3} B_{0}\right)^{5 / 8} t^{-3 / 4}$ & $\left(\sigma^{3} B_{0}\right)^{1 / 8} t^{1 / 4}$
\end{tabular}

\begin{tabular}{cc}
$B_{0}=0$ \\
\hline$e_{n} \propto$ & $l \propto$ \\
$B_{2}^{2 / 7} t^{-10 / 7}$ & $B_{2}^{1 / 7} t^{2 / 7}$ \\
$\left(\sigma B_{2}\right)^{3 / 8} t^{-5 / 4}$ & $\left(\sigma B_{2}\right)^{1 / 8} t^{1 / 4}$ \\
$\left(\sigma^{2} B_{2}\right)^{4 / 9} t^{-10 / 9}$ & $\left(\sigma^{2} B_{2}\right)^{1 / 9} t^{2 / 9}$ \\
$\left(\sigma^{3} B_{2}\right)^{1 / 2} t^{-1}$ & $\left(\sigma^{3} B_{2}\right)^{1 / 10} t^{1 / 5}$
\end{tabular}

TABLE 1. Scaling of the integrated energy $e_{n}$ and the integral scale $l$ for differing numbers of inhomogeneous directions

$$
\begin{array}{ccc}
n & \left\langle\boldsymbol{u}^{2}\right\rangle, B_{0} \neq 0 & \left\langle\boldsymbol{u}^{2}\right\rangle, B_{0}=0 \\
0 & B_{0}^{2 / 5} t^{-6 / 5} & B_{2}^{2 / 7} t^{-10 / 7} \\
1 & \left(\sigma B_{0}\right)^{1 / 3} t^{-4 / 3} & \left(\sigma B_{2}\right)^{1 / 4} t^{-3 / 2} \\
2 & \left(\sigma^{2} B_{0}\right)^{2 / 7} t^{-10 / 7} & \left(\sigma^{2} B_{2}\right)^{2 / 9} t^{-14 / 9} \\
3 & \left(\sigma^{3} B_{0}\right)^{1 / 4} t^{-3 / 2} & \left(\sigma^{3} B_{2}\right)^{1 / 5} t^{-8 / 5}
\end{array}
$$

TABLE 2. Scaling of the mean-square velocity at the origin for differing number of inhomogeneous directions

These decay laws were determined by Phillips (1956), who also considered the more complete problem of the final period of decay of the mean-square velocity at a point.

Predictions of high-Reynolds-number decay laws follow from assuming that $e_{n}$ scales on either $\sigma^{n} B_{0}$ or $\sigma^{n} B_{2}$, and $t$ alone, and is independent of the viscosity $v$. The dimensions of $e_{n}$ are $\left[e_{n}\right]=l^{2+n} t^{-2}$, the dimensions of the low-wavenumber spectral coefficients are given by $\left[B_{0}\right]=l^{5} / t^{2}$ and $\left[B_{2}\right]=l^{7} / t^{2}$, and $\sigma$ has units of length, so that from a dimensional analysis one obtains

$$
e_{n} \propto\left(\sigma^{n} B_{0}\right)^{(2+n) /(5+n)} t^{-6 /(5+n)} \quad \text { or } \quad e_{n} \propto\left(\sigma^{n} B_{2}\right)^{(2+n) /(7+n)} t^{-10 /(7+n)} .
$$

The explicit time dependence given in the first expression is expected to be exact because of the invariance of $B_{0}$ whereas the time dependence in the second expression can only be approximate since $B_{2}$ is an unknown function of time.

During the final period, all characteristic length scales of the flow field grow like $(v t)^{1 / 2}$ as a consequence of the viscous dissipation of the smallest scales and diffusion of the turbulence into the initial quiescent fluid. At high Reynolds numbers, we postulate that the length scale of the energetic eddies $l,(2.8)$, depends only on the low-wavenumber spectral coefficients and time $t$. The scaling law obtained from the above hypothesis applied to homogeneous turbulence has been shown to agree with the results of large-eddy simulations (Chasnov 1994). Thus, one determines dimensionally

$$
l \propto\left(\sigma^{n} B_{0}\right)^{1 /(5+n)} t^{2 /(5+n)} \quad \text { or } \quad l \propto\left(\sigma^{n} B_{2}\right)^{1 /(7+n)} t^{2 /(7+n)} .
$$

In table 1 , the above asymptotic laws for $e_{n}$ and $l$ are written explicitly for decaying turbulence with number of inhomogeneous directions $n=0,1,2,3$.

A Reynolds number of the flow field at time $t$ formed from $e_{n}, l$ and $v$ is given by $R(t)=e_{n}^{1 / 2} l^{1-n / 2} / v$, so that its scaling during the decay follows one of

$$
R(t)=\frac{\left(\sigma^{n} B_{0}\right)^{2 /(5+n)}}{v} t^{-(1+n) /(5+n)} \text { or } R(t)=\frac{\left(\sigma^{n} B_{2}\right)^{2 /(7+n)}}{v} t^{-(3+n) /(7+n)} .
$$


The Reynolds number of the flow field thus decreases during the decay in all cases so that the asymptotic laws above apply only over intermediate times for which the turbulence has become fully-developed and the Reynolds number can still be considered large.

Using the asymptotic decay laws for $e_{n}$ and $l$ given in table 1 , a similarity state for the spectrum $E_{n}(k, t)$ may be constructed from

$$
E_{n}(k, t)=e_{n} l \widehat{E}_{n}(\widehat{k}), \quad \widehat{k}=k l .
$$

Up to now, we have only considered statistics which are obtained by integrating over the inhomogeneous directions of the flow field so that a direct analogy could be made with known results for decaying homogeneous turbulence. We have thus avoided direct consideration of the transport of turbulence into quiescent fluid. Now we consider the evolution of the mean-square velocity of the fluid at a point. We postulate with somewhat less justification a self-similar decaying profile of the meansquare velocity as a function of the inhomogeneous coordinates $\boldsymbol{x}$ with origin at the centre of the initial disturbance:

$$
\left\langle\boldsymbol{u}^{2}\right\rangle(\boldsymbol{x}, t)=e_{n} d^{-n} U^{2}(\widehat{\boldsymbol{x}}), \quad \widehat{\boldsymbol{x}}=\boldsymbol{x} / \mathrm{d},
$$

where $d$ is a characteristic length scale of the width of the turbulent region within the infinite fluid and may be defined for instance by (2.10). The scaling of the self-similar profile on the integrated mean-square velocity $e_{n}$ is required by the equality of both sides of (3.8) after integration over the inhomogeneous directions.

The two length scales $l$ and $d$ are distinct $-l$ characterizes the size of the energetic eddies and $d$ the extent of the tubulent region of the fluid. The length scale $l$ is related to the energy spectrum $E_{n}(k)$ through (2.9), whereas there is no obvious relationship between $d$ and $E_{n}(k)$. Nevertheless, we may speculate that in an asymptotic similarity state all length scales of the flow field will grow at the same rate and $d$ will become proportional to $l$. If this is indeed the case, then it is possible to compute explicitly from (3.8) using (3.4) and (3.5) the scaling of the mean-square velocity at the origin of the initial disturbance:

$$
\left\langle\boldsymbol{u}^{2}\right\rangle \propto\left(\sigma^{n} B_{0}\right)^{2 /(5+n)} t^{-(6+2 n) /(5+n)} \quad \text { or } \quad\left\langle\boldsymbol{u}^{2}\right\rangle \propto\left(\sigma^{n} B_{2}\right)^{2 /(7+n)} t^{-(10+2 n) /(7+n)},
$$

depending on the form of the energy spectrum at small $k$. These decay laws are displayed for $n=0, \ldots, 3$ in table 2 ; the more rapid decay in the mean-square velocity at the origin with increasing number of inhomogeneous directions is due to the greater outward spatial transport of energy from regions of high turbulent activity towards regions of lower activity. For homogeneous turbulence $(n=0)$ there is no mean spatial transport of energy, $e_{0}=\frac{1}{2}\left\langle\boldsymbol{u}^{2}\right\rangle$, and the results in table 2 coincide with those in table 1.

We have thus postulated the existence of an asymptotic similarity state for decaying inhomogeneous turbulence, valid over intermediate times. This similarity state may not occur during the initial period of flow evolution if the integral scale of the turbulence $l$ and the extent of the spatial inhomogeneity $d$ are initially disparate so that their ratio is far from its equilibrium value. In particular, if $l \ll d$ then during the initial period of decay the vast central region of the flow field should approximate homogeneous turbulence. If $l \gg d$ initially, and with a single inhomogeneous direction, the initial turbulence will look two-dimensional on length scales smaller than $d$, and its dynamical evolution during the initial period may be quite different than that predicted above. Finally, at late times in the flow evolution, the Reynolds num- 
ber decreases asymptotically, eventually yielding the final period of decay solutions previously determined by Phillips (1956).

In the following Sections, we will present numerical simulations which test the scaling relations presented in tables 1 and 2 for $n=1$ corresponding to a single inhomogeneous direction, and the occurrence of an intermediate asymptotic similarity state for this flow field. With current computer capabilities, it is difficult to systematically study the effect of widely different initial values of the length scales $l$ and $d$ and instead we focus on the particular but important case when $l$ and $d$ are of comparable magnitude initially. It is not unreasonable to suppose that all initial conditions of the flow field will approach this state asymptotically at large Reynolds numbers provided the similarity states determined above occur, and we will offer some limited numerical evidence that this is indeed the case.

\section{Numerical simulation method}

\subsection{Basics}

We wish to perform numerical simulations of decaying inhomogeneous turbulence in an infinite domain to test the scaling relations determined in the previous Section. Although in this work we will only present results from simulations with a single inhomogeneous direction, we nevertheless develop the general numerical methodology for simulating one to three inhomogeneous directions. For the directions in which the turbulence is homogeneous, it is common to apply periodic boundary conditions to the flow field and to adjust the initial integral scale of the turbulence to be much less than the periodicity length. For decaying turbulence in which the integral scale increases during the decay, the evolution time of the flow field must be less than the time at which the integral scale becomes comparable to the periodicity length. Further evolution of the flow field past this time would result in unphysical results which depend on the artificial periodicity length.

For directions in which the turbulence is inhomogeneous such that velocity fluctuations vanish at infinity, we can still apply periodic boundary conditions within a finite subdomain, and choose initial conditions such that the velocity fluctuations are negligible on the boundaries. Such a numerical method may not be the most accurate nor efficient (for another approach, see Corral \& Jimenez 1995), but it is the most expedient since it is the easiest to implement with our present pseudo-spectral code which applies periodic boundary conditions in all three directions. A triply periodic domain was also used in a recent numerical simulation of the shearless turbulence mixing layer (Briggs et al. 1996). We thus define the Fourier transform of our velocity field as

$$
\boldsymbol{u}(\boldsymbol{x})=\sum_{n_{x}, n_{y}, n_{z}} \widehat{\boldsymbol{u}}(\boldsymbol{k}) \exp (\mathrm{i} \boldsymbol{k} \cdot \boldsymbol{x})
$$

with

$$
\boldsymbol{k}=\left(\frac{2 \pi}{L_{x}} n_{x}, \frac{2 \pi}{L_{y}} n_{y}, \frac{2 \pi}{L_{z}} n_{z}\right),
$$

where $L_{x}, L_{y}$, and $L_{z}$ are the periodicity lengths in the three directions, $n_{x}, n_{y}$, and $n_{z}$ are integers, and the Fourier components of the velocity field satisfy the complex-conjugate symmetry relation $\widehat{\boldsymbol{u}}(-\boldsymbol{k})=\widehat{\boldsymbol{u}}(\boldsymbol{k})^{*}$.

Let us denote periodicity lengths in homogeneous directions to be equal to $L$ and 
in inhomogeneous directions to be equal to $D$. The spectrum $E_{n}(k)$ is computed from

$$
E_{n}(k)=\frac{2 \pi D^{n} k^{2}}{S_{k}} \sum_{k-(\delta / 2) \leqslant|q| \leqslant k+(\delta / 2)} \widehat{u}_{i}(\boldsymbol{q}) \widehat{u}_{i}(-\boldsymbol{q})
$$

where $S_{k}$ is the number of Fourier modes within a spherical wavenumber shell of width $\delta$ centred at $k$. Typically, we choose $\delta=2 \pi / D$.

The mean-square turbulence velocity distribution is computed by inverse Fourier transforming the velocity field in the inhomogeneous directions. The $N$ physical space collocation points in each inhomogeneous direction (with generic coordinate $\xi$ ) are distributed according to

$$
\xi_{j}=D\left(\frac{j}{N}-\frac{1}{2}\right), \quad j=1, \ldots, N .
$$

For instance, with a single inhomogeneous direction, say $y$, the mean-square velocity at each collocation point $y_{j}$ is given by

$$
\left\langle\boldsymbol{u}^{2}\right\rangle\left(y_{j}\right)=\sum_{n_{x}, n_{z}} \widehat{u}_{i}\left(k_{x}, y_{j}, k_{z}\right) \widehat{u}_{i}\left(-k_{x}, y_{j},-k_{z}\right),
$$

where here $\widehat{u}_{i}$ denotes the Fourier component of the velocity field with transform in $x$ and $z$ only.

The integrated mean-square velocity of the turbulence is computed from the threedimensional Fourier components using

$$
e_{n}=\frac{1}{2} D^{n} \sum_{n_{x}, n_{y}, n_{z}} \widehat{u}_{i}(\boldsymbol{k}) \widehat{u}_{i}(-\boldsymbol{k}) .
$$

The integral scale $l,(2.8)$, is computed using (Chasnov 1994)

$$
l=\frac{\pi D^{n}}{4 e_{n}}\left[\frac{L}{\pi}|\widehat{\boldsymbol{u}}(0,0,0)|^{2}+\sum_{\boldsymbol{k} \neq 0} \frac{|\widehat{\boldsymbol{u}}(\boldsymbol{k})|^{2}}{k}\left(\frac{2}{\pi} \operatorname{Si}(k L / 2)\right)\right],
$$

where $\operatorname{Si}(x)$ is a sine integral, and the integration over the separation length $r$ in (2.8) has been taken from zero to $L / 2$. The length scale $d$, (2.10), characteristic of the spatial inhomogeneity may be computed by inverse Fourier transforming the velocity field in the inhomogeneous directions, forming the product with $|\boldsymbol{x}|^{2}$ on the physical space collocation points given by (4.4), and then summing over all the grid points in the inhomogeneous directions to perform the integration in physical space directly. The average over the homogeneous directions may be taken in Fourier space in the usual way.

An important non-dimensional statistic will be the ratio of the two length scales $d$ and $l$ :

$$
r(t)=d(t) / l(t),
$$

and we expect that if a complete similarity state in both the spectrum and mean-square velocity distribution occurs, $r$ approaches a constant of order unity asymptotically in time. Furthermore, the constants introduced in defining $d,(2.10)$, have been chosen so that the maximum value of $d$, attained when the turbulence approaches homogeneity in the defined inhomogeneous directions, is $D / 2$ and (with $D=L$ ) is equal to the maximum value of the integral scale $l$ which occurs when the flow field consists of only a mean velocity (a single Fourier mode with zero wavenumber). 
The attainment of sufficiently high Reynolds numbers so that molecular viscosity does not explicitly enter the scaling relations necessitates the use of large-eddy simulations with an ad-hoc subgrid-scale model. Several choices of subgrid-scale model are possible, and we choose the simplest to implement within a spectral code with the hope that the results for the decay exponents will be relatively insensitive to our choice of model. The subgrid model we use is a generalization of the Kraichnan, Chollet-Lesieur model (Kraichnan 1976; Chollet \& Lesieur 1981) with a scaling of the subgrid eddy viscosity on $E_{n}\left(k_{m}, t\right)$ and $k_{m}$, where $k_{m}$ is the maximum wavenumber magnitude carried in the spectral simulation. Dimensionally, one has

$$
v_{e}(k, t)=v_{e}^{+}\left(k / k_{m}\right)\left(k_{m}^{n-1} E_{n}\left(k_{m}, t\right)\right)^{1 / 2},
$$

and we take the same form of the dimensionless eddy viscosity $v_{e}^{+}$as in earlier work (Chasnov 1994). This model was originally proposed for homogeneous turbulence simulation and its use in inhomogeneous flows is questionable since the eddy viscosity has the same value in both actively turbulent regions and quiescent fluid. Physically, the eddy viscosity should approach zero in regions where the fluid is at rest. A subgrid model such as the dynamic model (Germano et al. 1991) could have better properties in this regard since the eddy viscosity is a function of the inhomogeneous coordinate with value set by the turbulence itself, but this model is somewhat more difficult to implement and we do not make use of it here.

\subsection{Initial conditions}

We will specify the statistical properties of our random initial conditions at $t=0$ by the spectrum $E_{n}(k)$ and the averaged mean-square velocity of the turbulence $\left\langle\boldsymbol{u}^{2}\right\rangle(\boldsymbol{x})$ as a function of the inhomogeneous coordinates $\boldsymbol{x}$. The spectrum and mean-square velocity are related by the integral relation

$$
e_{n}=\int_{0}^{\infty} E_{n}(k) \mathrm{d} k=\frac{1}{2} \int\left\langle\boldsymbol{u}^{2}\right\rangle(\boldsymbol{x}) \mathrm{d} x^{n} .
$$

We choose as our initial spectrum

$$
E_{n}(k)=\frac{1}{2} a_{s} \sigma^{n} u_{0}^{2} k_{p}^{-1}\left(\frac{k}{k_{p}}\right)^{s} \exp \left[-\frac{s k^{2}}{2 k_{p}^{2}}\right],
$$

with $s$ an even natural number and where $k_{p}$ is the wavenumber at which $E_{n}(k)$ is maximum. The constant $a_{s}$ is determined so that

$$
e_{n}=\frac{1}{2} \sigma^{n} u_{0}^{2},
$$

yielding

$$
a_{s}=\left(\frac{2}{\pi}\right)^{1 / 2} \frac{s^{(s+1) / 2}}{1 \times 3 \cdots(s-1)} .
$$

Furthermore, we choose a Gaussian profile for our initial mean-square velocity statistic satisfying the constraint given by (4.10):

$$
\left\langle\boldsymbol{u}^{2}\right\rangle(\boldsymbol{x})=\frac{1}{(2 \pi)^{n / 2}} u_{0}^{2} \exp \left[-\frac{|\boldsymbol{x}|^{2}}{2 \sigma^{2}}\right],
$$

where $\boldsymbol{x}$ is the coordinate in the inhomogeneous direction. 
The length scales $l$ and $d$ defined in (2.9) and (2.10) may be computed at the initial instant of time using (4.11) and (4.14). One finds

$$
l_{0}=\left(\frac{\pi}{2 s}\right)^{1 / 2} \frac{2 \times 4 \cdots s}{1 \times 3 \cdots(s-1)} k_{p}^{-1}, \quad d_{0}=\frac{\sqrt{3}}{2} \sigma
$$

so that as expected $l_{0}$ and $d_{0}$ are directly proportional to $k_{p}^{-1}$ and $\sigma$, respectively. The ratio $r=d / l$ of these length scales at the initial instant for the particular cases $s=2$ and 4 takes the approximate values

$$
r_{0} \approx \begin{cases}0.4886 \sigma k_{p} & \text { if } s=2 \\ 0.5182 \sigma k_{p} & \text { if } s=4\end{cases}
$$

An initial flow field is generated with the above energy spectrum and mean-square velocity profile through an iterative process. To begin the interation procedure a random velocity field is generated in Fourier space with spectrum given by (4.11) following the method of Rogallo (1981), i.e. we define

$$
f_{1}(\boldsymbol{k})=\left(\frac{E_{n}(k)}{2 \pi D^{n} k^{2}}\right)^{1 / 2} \exp \left(\mathrm{i} 2 \pi \theta_{1}\right) \cos 2 \pi \phi, \quad f_{2}(\boldsymbol{k})=\left(\frac{E_{n}(k)}{2 \pi D^{n} k^{2}}\right)^{1 / 2} \exp \left(\mathrm{i} 2 \pi \theta_{2}\right) \sin 2 \pi \phi
$$

where $\theta_{1}, \theta_{2}$, and $\phi$ are uniform deviates chosen independently for each $\boldsymbol{k}$ subject to the complex-conjugate symmetry of the Fourier components of the velocity field. The three components of a random velocity field which satisfies the continuity equation are then constructed using $f_{1}$ and $f_{2}$ by

$$
\widehat{\boldsymbol{u}}^{(0)}=\frac{1}{k k_{\perp}}\left(f_{2} k_{x} k_{y}-f_{1} k k_{z},-f_{2} k_{\perp}^{2}, f_{1} k k_{x}+f_{2} k_{y} k_{z}\right),
$$

where $k=\left(k_{x}^{2}+k_{y}^{2}+k_{z}^{2}\right)^{1 / 2}$ and $k_{\perp}=\left(k_{x}^{2}+k_{z}^{2}\right)^{1 / 2}$.

In the first step of our two-step iteration procedure, the velocity field is inverse Fourier transformed to physical space in the inhomogeneous directions and its magnitude at each inhomogeneous coordinate position is adjusted so that the meansquare velocity follows (4.14) exactly. The velocity field is then Fourier transformed back to wave space. In the second step of the procedure, the Fourier components of the velocity field are projected onto a divergence-free field, and their amplitudes at each Fourier mode are adjusted so that the spectrum $E_{n}(k)$ satisfies (4.11) exactly.

To be precise, for a single direction of inhomogeneity $(n=1)$ the first step of the interation procedure is the assignment for each wavenumber component $k_{x}, k_{z}$ in the homogeneous directions and for each physical space collocation point $y_{j}$ in the inhomogeneous direction,

$$
\widehat{u}_{i}^{(m+1 / 2)}\left(k_{x}, y_{j}, k_{z}\right)=\left[\frac{\left\langle\boldsymbol{u}^{2}\right\rangle\left(y_{j}\right)}{\left\langle\boldsymbol{u}^{2}\right\rangle^{(m)}\left(y_{j}\right)}\right]^{1 / 2} \widehat{u}_{i}^{(m)}\left(k_{x}, y_{j}, k_{z}\right),
$$

where $\left\langle\boldsymbol{u}^{2}\right\rangle(y)$ is the target mean-square velocity distribution given by $(4.14),\left\langle\boldsymbol{u}^{2}\right\rangle^{(m)}(y)$ is the actual mean-square velocity distribution at the $m$ th stage of the iteration process, and the caret denotes the Fourier component with transform in $x$ and $z$ only. The average is taken over the homogeneous directions in $x$ and $z$. 


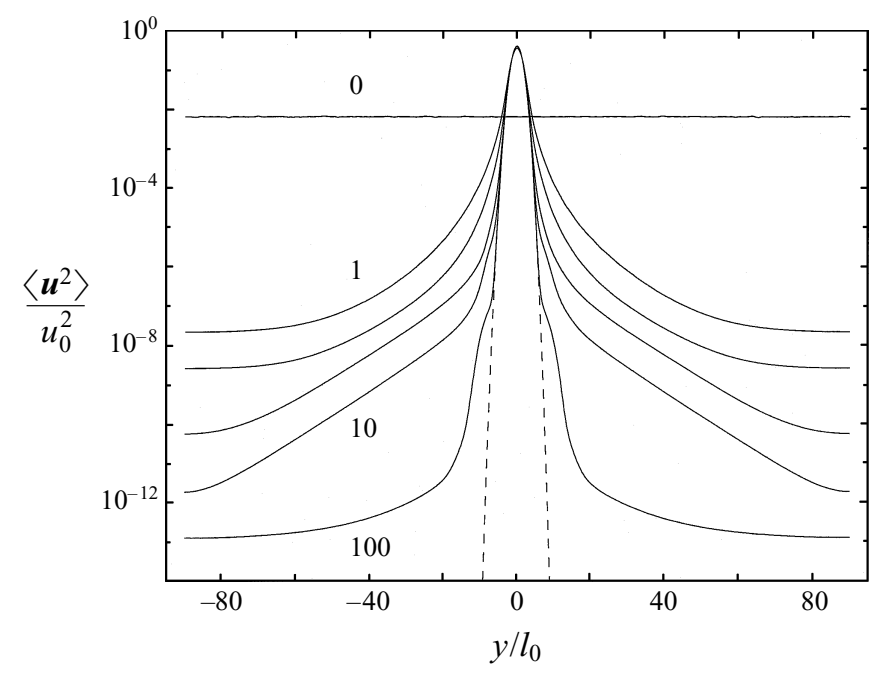

FIGURE 1. Convergence of the numerical results (solid lines) to the specified initial mean-square velocity distribution (dashed line). Iterations shown are $0,1,2,5,10$ and 100.

The second step of the iteration process is the assignment for each $\left(k_{x}, k_{y}, k_{z}\right)$

$$
\widehat{u}_{i}^{(m+1)}(\boldsymbol{k})=\left[\frac{E_{n}(k)}{2 \pi D^{n} k^{2}\left|\widehat{P}_{i j}(\boldsymbol{k}) \widehat{u}_{j}^{(m+1 / 2)}(\boldsymbol{k})\right|^{2}}\right]^{1 / 2} P_{i j}(\boldsymbol{k}) \widehat{u}_{j}^{(m+1 / 2)}(\boldsymbol{k}),
$$

where $E_{n}(k)$ is the target spectrum, and $P_{i j}(\boldsymbol{k})=\delta_{i j}-k_{i} k_{j} / k^{2}$ projects the velocity field onto its divergence-free part.

The mean velocity of the fluid, corresponding to the zero-wavenumber mode, must be treated as a special case in (4.20). The mean velocity is non-zero only when $s=2$ in (4.11) for which it takes the squared value

$$
|\widehat{\boldsymbol{u}}(0)|^{2}=\left(\frac{\sigma}{D}\right)^{n} \frac{u_{0}^{2}}{\pi^{3 / 2} k_{p}^{3}} .
$$

We have chosen not to assign the zero-wavenumber mode in (4.20); nevertheless it was observed to converge to its theoretical value for flows with a single inhomogeneous direction.

The procedure for determining the initial flow field is iterated until convergence of the mean-square velocity profile after application of (4.20). The convergence of the above procedure for a single inhomogeneous direction is illustrated in figure 1 . The initial energy spectrum is given by (4.11) with $n=1, s=2, u_{0}=1, k_{p}=32$, and resolution $256 \times 512 \times 256$. The target mean-square velocity distribution is given by (4.14) with $\sigma=0.06396$, so that $r_{0}=1$ in (4.16). The curves show the convergence of the distribution after 100 iterations. The dashed curve is the analytical form of the mean-square velocity profile given by (4.14). The mean-square velocity profile rapidly converges to the analytical profile in the centre of the domain, but converges much more slowly at the tails.

With our initial conditions of the flow field specified, the only non-dimensional parameters which characterize our initial statistical state are the form of the lowwavenumber spectrum specified by $s$, which we take to be either 2 or 4 to test the 
scalings of $\S 3$, and the ratio $r_{0}$ of the initial length scales, for which simulations are performed with values 1,2 , and 4 .

\section{Numerical results for a single inhomogeneous direction}

\subsection{Test of accuracy}

The largest errors in our numerical simulations are due to inadaquate small-scale resolution and the assumption of periodic boundary conditions in both the homogeneous and inhomogeneous directions. The means of reducing these two types of errors are in conflict: improving the resolution requires positioning the initial energy distribution at the largest scales whereas reducing the effect of boundary conditions requires just the opposite. Obviously, a compromise is necessary and it is prudent to perform a simple test to judge the overall accuracy of our calculations.

Our main simulation which tests the scaling laws of $\S 3$ for $s=2$, corresponding to a $k^{2}$ spectrum at low wavenumbers, is performed with $256 \times 512 \times 256$ grid points and $k_{p}=32, u_{0}=1$ in (4.11), and with $r_{0}=1$. The grid spacing is the same in each direction so that the box length in the single inhomogeneous direction is twice that of the homogeneous directions (we take $D=2^{5 / 3} \pi ; L=2^{2 / 3} \pi$ ). The accuracy of this calculation is judged by performing two additional simulations with $128 \times 256 \times 128$ grid points and $k_{p}=32$ or $k_{p}=16$. The former calculation $\left(k_{p}=32\right)$ quantifies the subgrid-scale errors since $k_{p} / k_{m}$ is reduced by a factor of 2 , where $k_{m}$ is the maximum resolved wavenumber of the computation. The latter calculation $\left(k_{p}=16\right)$ measures the influence of the periodic boundary conditions on the results by reducing $k_{p} / k_{0}$ by a factor of 2 , where $k_{0}$ is the minimum wavenumber of the simulation, while keeping $k_{p} / k_{m}$ fixed.

The decay of the integrated mean-square velocity and the time evolution of the length scales $l$ and $d$ for these three calculations are shown in figure 2. The solid line are the results of the higher resolution calculation; the dashed line and dotted lines are the lower resolution calculations. The dotted line tests the subgrid-scale model errors $\left(k_{p}=32\right)$ and the dashed line tests the errors due to the periodic boundary conditions $\left(k_{p}=16\right)$. The large differences between the dotted lines on the one hand, and the dashed and solid lines on the other, at the early times of evolution indicates substantial errors due to the inadaquate small-scale resolution. However these errors diminish at later times after the integral scale of the flow has increased significantly, thus providing additional small-scale resolution relative to the energy-containing scales. The improvement in accuracy of a large-eddy simulation after long times of decay has already been observed in decaying isotropic turbulence simulations (Piomelli \& Chasnov 1996). Differences between the solid and dashed lines at the latest times of evolution indicate either poor statistical sample of the largest eddies, or an adverse influence of the periodic boundary conditions. It is possible to rectify a poor statistical sample by averaging over an ensemble of flows (which we do not attempt here), but errors due to the direct influence of the periodic boundary conditions are more serious. However, the calculations shown in figure 2 demonstrate that the magnitude of these errors is reasonably small and may be acceptable over the times simulated. We further comment that the calculation of $e_{n}$ appears to be more robust than that of the length scales.

\subsection{Main results}

We now present results to directly test the scaling laws of $\S 3$. The calculations we present in detail are of resolution $256 \times 512 \times 256$, with $k_{p}=32$ and $u_{0}=1$ in (4.11). 

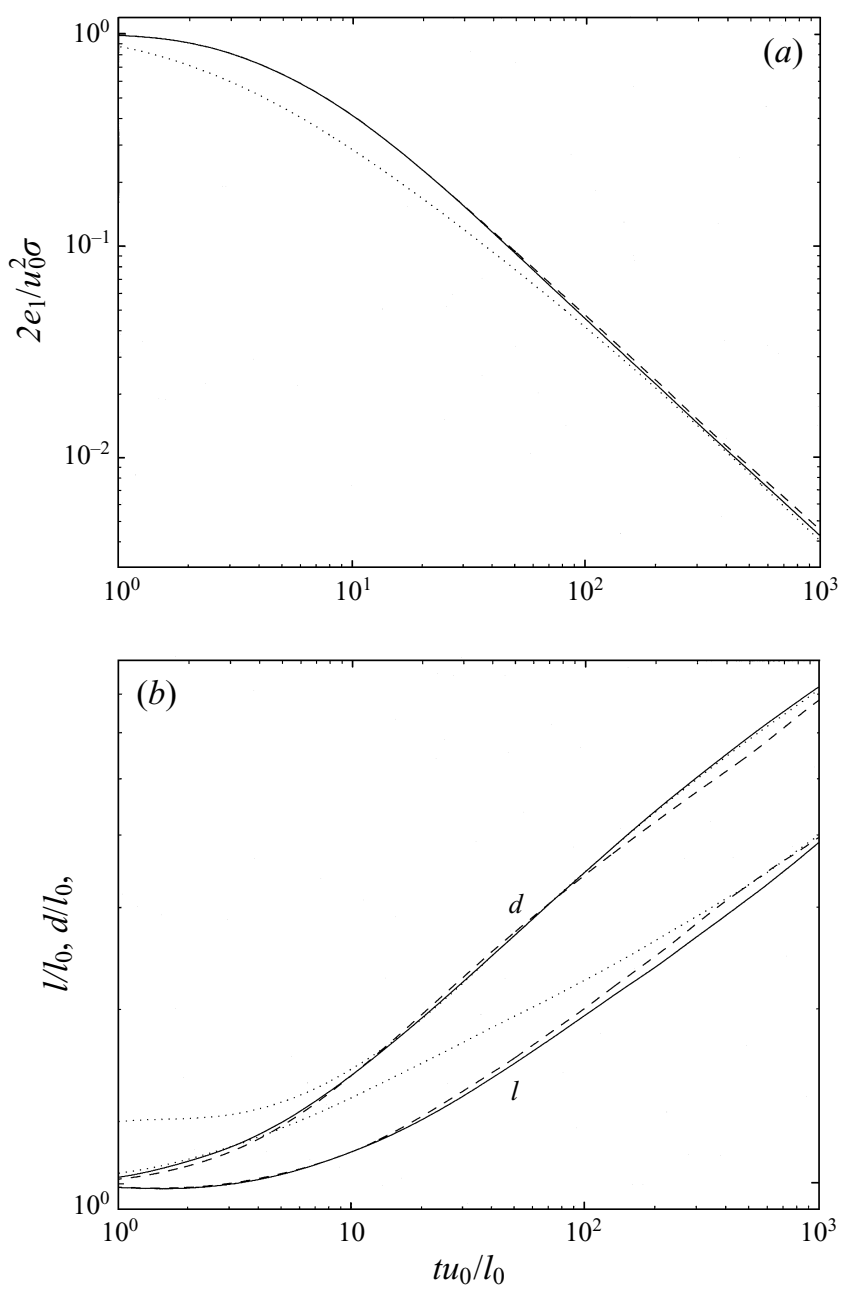

FIGURE 2. Test of the effects of small-scale resolution (dotted line) and periodic boundary conditions (dashed line). The solid line is the most accurate calculation to which the other two should be compared. (a) Time-evolution of the integrated mean-square velocity $e_{1}$. (b) Time-evolution of the inhomogeneity length scale $d$ and the integral scale $l$.

Both scaling laws of $\S 3$ are tested by performing two calculations, the first with $s=2$ in (4.11), and the second with $s=4$. Furthermore, we choose $\sigma$ according to (4.16) so that $r_{0}=1$ in the first simulation and $r_{0}=2$ in the second. The latter choice has been determined to more rapidly approach the asymptotic similarity state.

In figure 3, we plot from both of the above simulations the logarithmic derivative of the integrated mean-square velocity $e_{1}$, which corresponds to the power-law exponent of the time if the decay proceeds as a power law. The long-time results from the numerical calculations are to be compared to the explicit power-law exponents of the time found in table 1 of $\S 3$ with $n=1$, corresponding to a single inhomogeneous direction. These analytical results are shown as the dotted lines in figure 3 . Our calculations of the power-law exponent of $e_{1}$ with $s=2$ labelled by $B_{0}$ yields an asymptotic decay exponent fluctuating between -1.02 and -1.03 , which deviates at most by $3 \%$ from the theoretical exponent -1 . The decay exponent of $e_{1}$ for $s=4$ labelled by $B_{2}$ is approximately -1.17 at the latest time calculated, which is $7 \%$ 


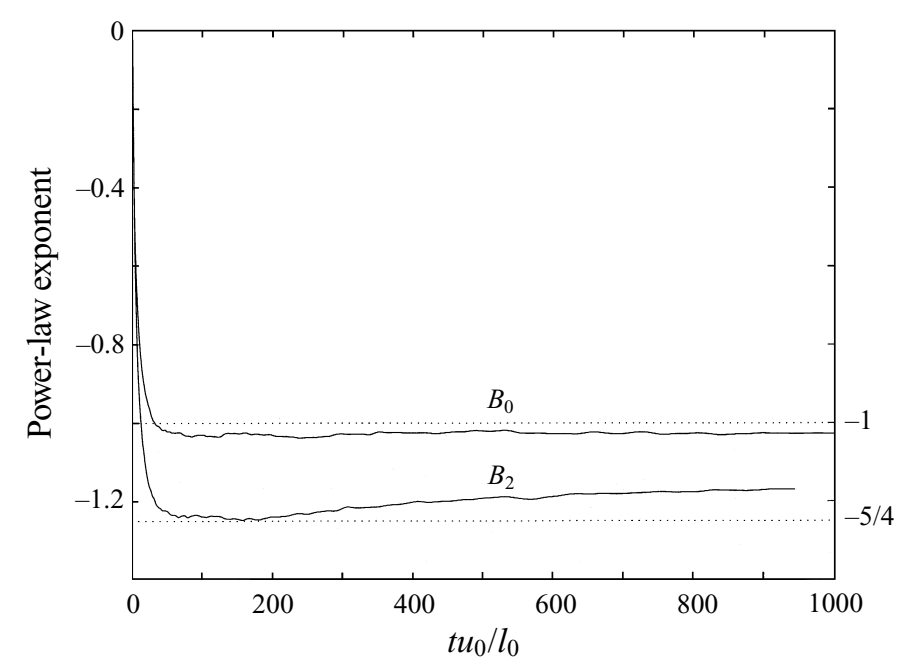

FIGURE 3. Time evolution of the power-law exponent of $e_{1}$. The solid lines labelled with the non-zero leading-order low-wavenumber spectral coefficient are the results of the large-eddy simulations and the dotted lines are the exact and approximate analytical results discussed in $\S 3$.

smaller in magnitude than the theoretical decay exponent -1.25 , obtained under the (false) assumption that $B_{2}(t)$ is independent of time. An increase in $B_{2}(t)$ during the decay results theoretically in a decay exponent smaller in magnitude than -1.25 , as is found from the simulation data.

In figure 4 the logarithmic derivatives of the length scales $d$ and $l$ from both calculations are plotted, and the dotted lines again correspond to the theoretical results. Here, the solid line corresponds to $s=2$ and the dashed lines to $s=4$, and the lines are labelled by the corresponding length scales $d$ and $l$. The agreement between the simulations and the theoretical results are not as good as in figure 3, and we will later show in $\S 5.3$ that this is due to adverse affects of assuming periodic boundary conditions. Nevertheless, the qualitative agreement exhibited here is not unreasonable.

As discussed in $\$ 3$, a complete similarity state for both the spectrum and the distribution of the mean-square velocity based on the low-wavenumber coefficients requires the ratio of the length scales $r(t)=d / l$ to approach a constant asymptotically. A study of the evolution of $r(t)$ for $s=2$ and 4 and for $r_{0}=1,2$ and 4 is presented in figure 5 . The additional computations presented are also of resolution $256 \times 512 \times 256$ with $k_{p}=32$ and $u_{0}=1$. The desired value of $r_{0}$ is obtained by changing $\sigma$. Although the numerical results are not decisive, they do suggest that the flow field evolves in time so as to obtain nearly the same constant value of $r$ asymptotically during the decay. The results of figure $5(a)$ point to a unique intermediate asymptotic similarity state of the flow field when $s=2$, independent of all aspects of the initial conditions except the initial value of the low-wavenumber spectral coefficient $B_{0}$. However, when $s=4$ the precise value of $B_{2}(t)$ during the decay must depend on its time history so that strictly speaking a unique similarity state does not occur, though it is still plausible that $r(t)$ approaches a unique value during the intermediate similarity state for all initial conditions.

We consider next the time evolution of the spectrum $E_{1}(k, t)$, presented in figure 6 . Figure 6(a) corresponds to $s=2$ and figure $6(b)$ to $s=4$. The non-dimensional times plotted are shown in the caption. The invariance of the low-wavenumber coefficient 


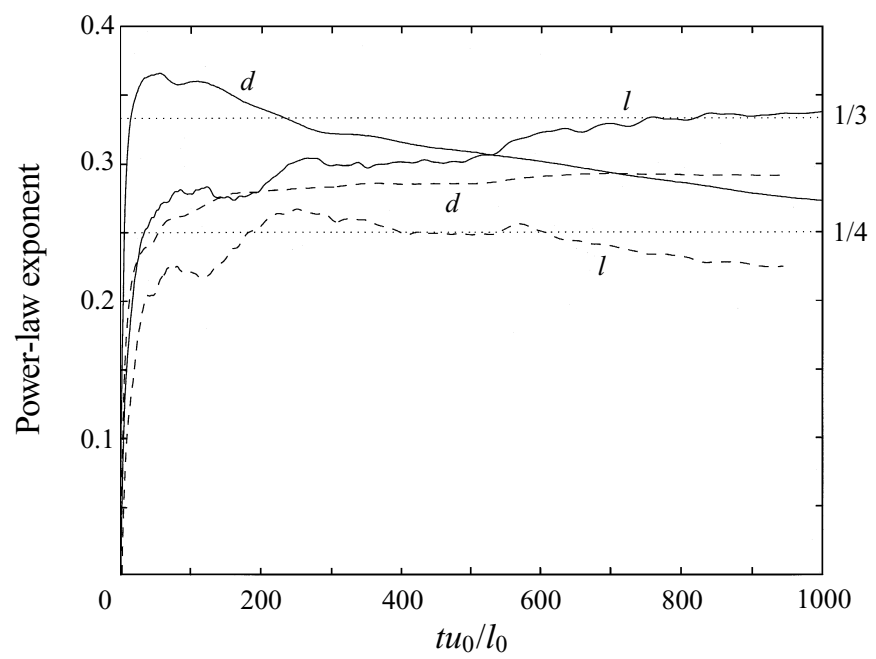

FIGURE 4. Time evolution of the power-law exponents of the length scales $d$ and $l$. The solid lines correspond to $s=2$ and the dashed lines to $s=4$. The lines are labelled by $d$ and $l$ and correspond to the logarithmic derivatives of these length scales. The dotted lines are the exact and approximate analytical results discussed in $\S 3$
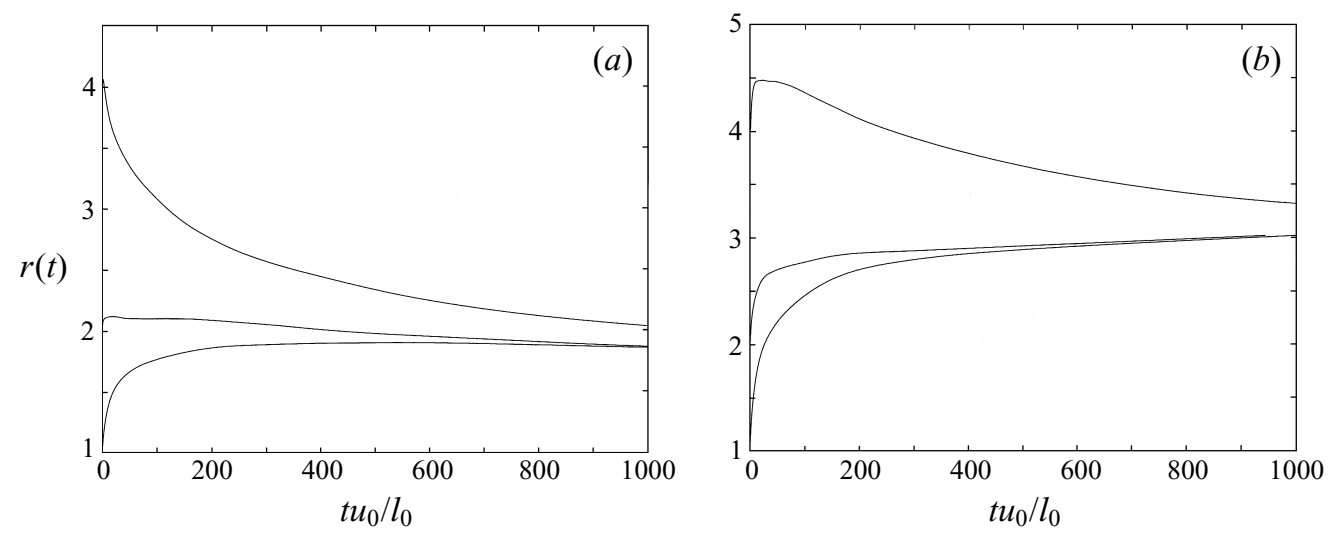

FIGURE 5. Time evolution of the ratio of the length scales $r(t)=d(t) / l(t)$ for initial values $r_{0}=1,2$ and 4 . (a) $s=2 ;(b) s=4$.

of the spectrum for $s=2$ is apparent in figure $6(a)$, whereas the slow increase in the low-wavenumber spectral coefficient $B_{2}(t)$ can be observed in figure $6(b)$. The evolution of these spectra appear similar to previous numerical results obtained for the decay of homogeneous turbulence (Chasnov 1994). A collapse of the spectra in self-similar variables, see (3.7), at the latest times simulated is displayed in figures 7(a) and $7(b)$. For $s=2$, the spectrum $t^{2 / 3} E_{1}$ is plotted versus $t^{1 / 3} k$, and for $s=4, t E_{1}$ is plotted versus $t^{1 / 4} k$. The times, spectra, and wavenumbers are non-dimensionalized using $u_{0}$ and $l_{0}$. The collapse of the spectra is reasonable at low wavenumbers and near the spectral peaks, but not as good at large wavenumbers. This may be indicative of subgrid-scale modelling errors.

Finally, the time evolution of the distribution of the mean-square velocity, $\left\langle\boldsymbol{u}^{2}\right\rangle(y, t)$, is presented in figures $8(a)$ and $8(b)$ corresponding to $s=2$ and $s=4$, respectively. The times plotted are the same as those plotted for the spectra. A transport of 

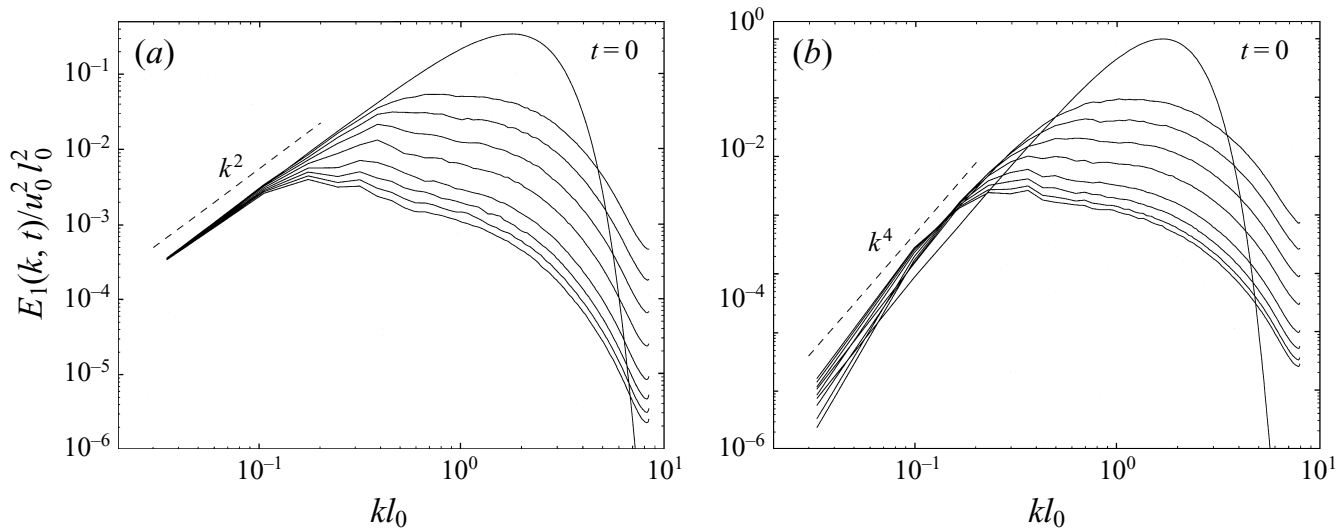

FIgURE 6. Time evolution of the spectrum $E_{1}(k, t)$. The times plotted correspond to $t u_{0} / l_{0}=0,25$,

$50,100,200,400,600,800$, and 1000 in $(a)$ and 943 instead of 1000 in $(b) .(a) s=2 ;(b) s=4$.
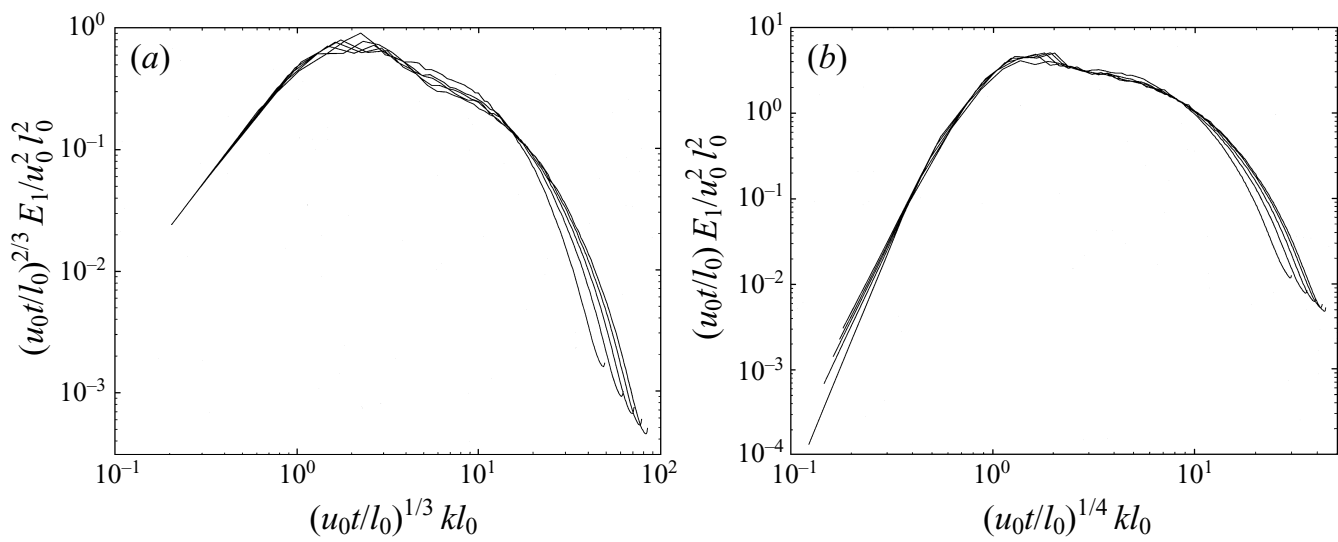

FIGURE 7. Rescaling of the spectra at the last five times plotted in figure 6 according to the similarity state predicted in $\S 3$. (a) $s=2 ;(b) s=4$.

the energy during the decay outward along the inhomogeneous directions is readily apparent from the figures. The rather rapid initial increase in the energy at the ends of the computational box during the decay is due to the loss of small-scale resolution as the energy cascades to the largest computational wavenumbers. At the last times calculated, the value of the mean-square velocity at the ends of the computational box are still approximately six orders of magnitude less than that at the centre, and it seems reasonable that our periodic box still approximates an infinite domain. The corresponding self-similar mean-square velocity profiles, (3.9), are plotted in figures $9(a)$ and $9(b)$. For $s=2$, the distribution $t^{4 / 3}\left\langle\boldsymbol{u}^{2}\right\rangle$ is plotted versus $t^{-1 / 3} y$, and for $s=4, t^{3 / 2}\left\langle\boldsymbol{u}^{2}\right\rangle$ is plotted versus $t^{-1 / 4} y$. A good collapse of the profiles is observed. The self-similarity of the mean-square velocity distribution is best at the centre of the initial tubulent fluid, and deteriorates at the ends, presumably due to adverse influences of the periodic boundary conditions.

\subsection{Additional computations}

The results just presented give us some confidence in the overall correctness of the theoretical scaling relations determined in $\$ 3$. The decay laws of the integrated mean- 

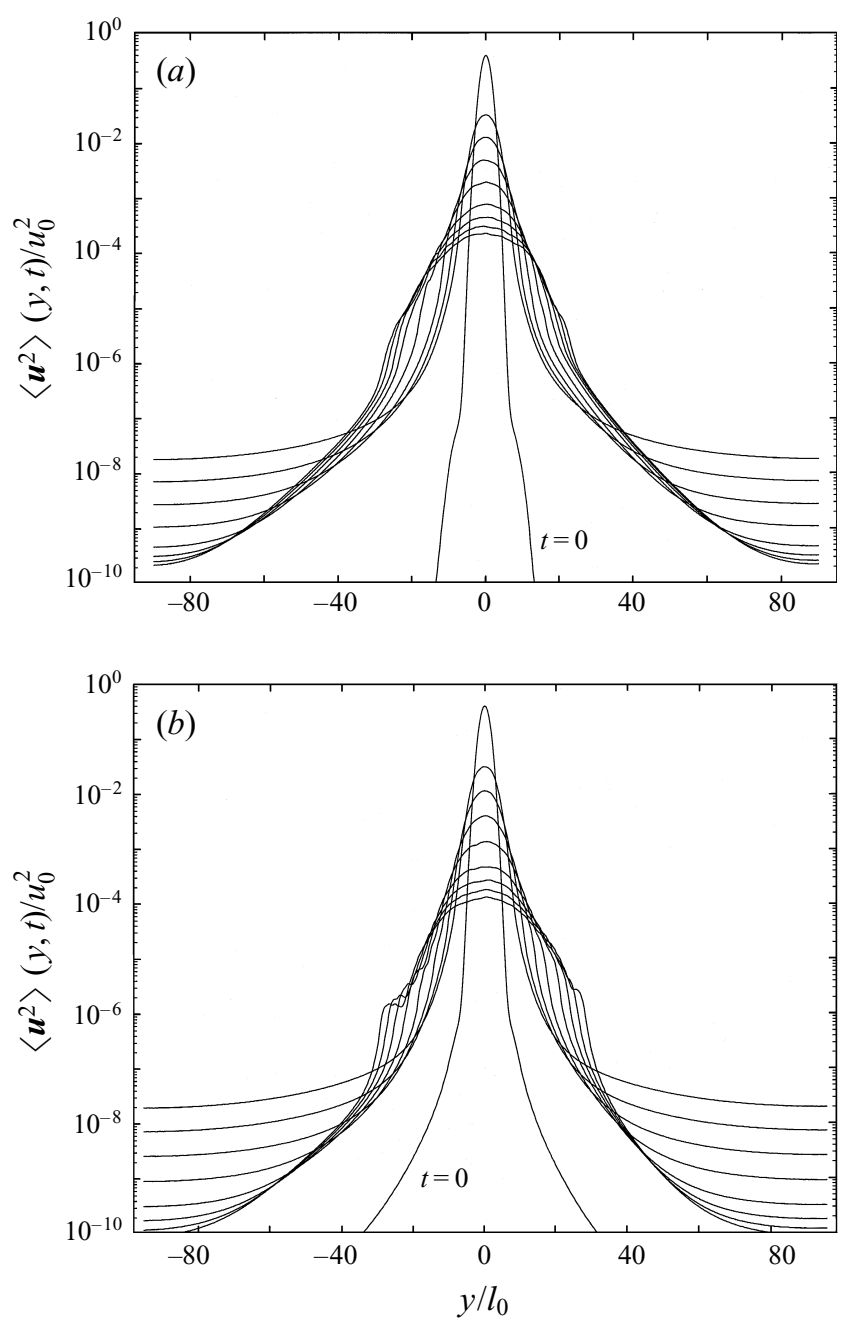

FIGURE 8 . Time evolution of the mean-square velocity distribution $\left\langle\boldsymbol{u}^{2}\right\rangle(y, t)$. The times plotted are the same as in figure 6. (a) $s=2 ;(b) s=4$.

square velocity are in good agreement with the analytical decay laws, and the spectra and mean-square velocity distributions appear to decay following the predicted selfsimilar forms. The main lack of agreement between the simulation results and the theoretical scaling laws is in the time exponents of the integral scales, though we have already provided evidence that the computation of these exponent values are the most susceptible to the numerical errors introduced by the use of periodic boundary conditions.

To try to minimize the adverse consequences of the periodic boundary conditions, we have performed additional simulations with $256 \times 512 \times 256$ grid points with the initial spectral peak at a very large wavenumber $\left(k_{p}=126\right)$. As we have already shown at the beginning of this Section, these calculations are inaccurate over short simulation times, but increase in accuracy as the spectral peak moves to smaller wavenumbers during the decay. The calculation results we reproduce here were performed with $s=2$ and with $r_{0}=1$ and 4 , with the main goal to accurately 

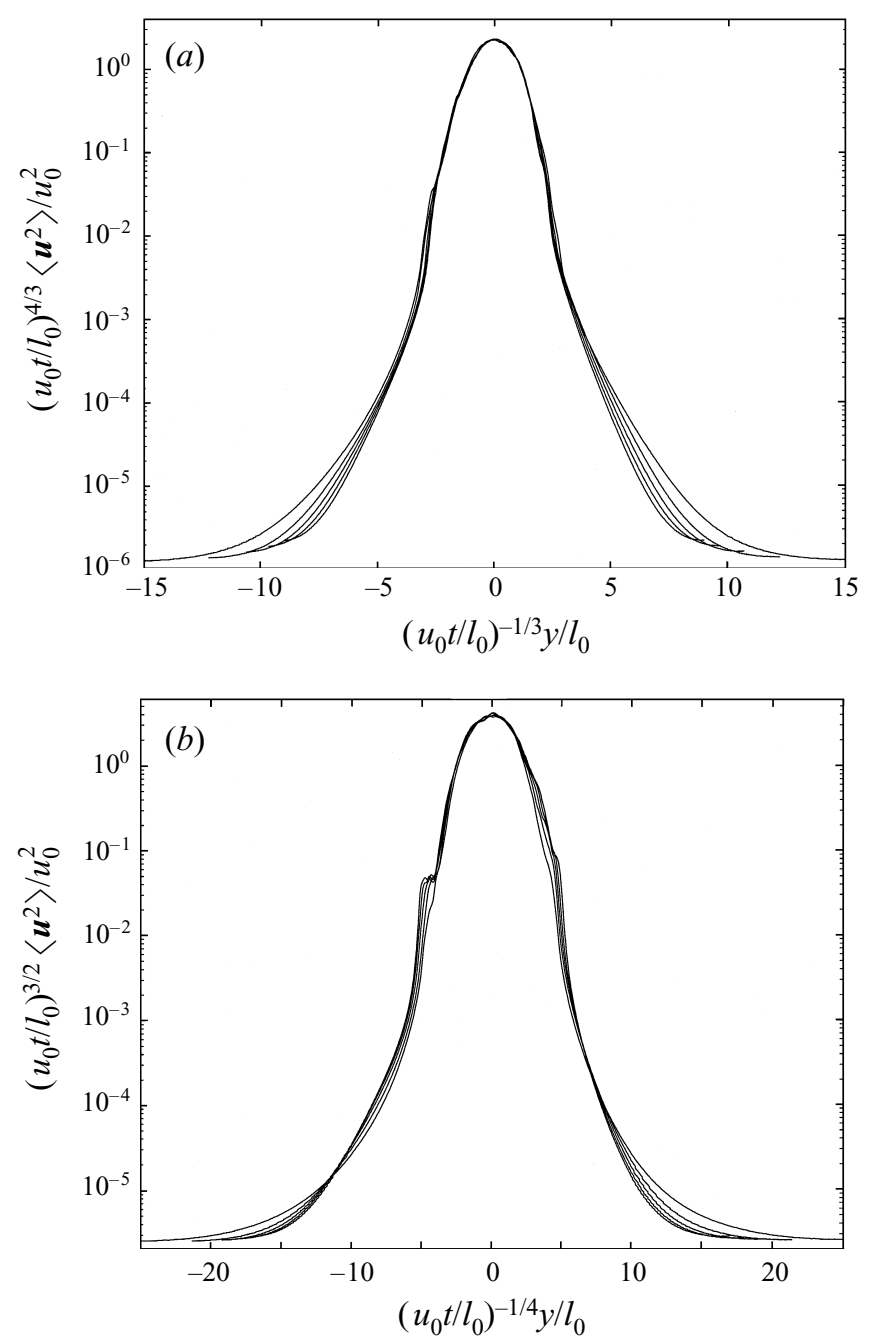

FiguRE 9. Rescaling of the mean-square velocity distribution at the last five times plotted in figure 8 according to the similarity state predicted in $\S 3$. (a) $s=2 ;(b) s=4$.

compute the long-time power-law exponents of the integral scales so as to precisely test the theoretical result for this exponent found in $\$ 3$.

In figure 10, the logarithmic derivative of the length scales $d$ and $l$ from these two calculations are plotted versus time. For $r_{0}=1$, figure 10(a), the power-law exponent of $d$ is now found to be in excellent agreement asymptotically with the predicted value $1 / 3$, shown as the dotted line, while the power-law exponent of $l$ is observed to be slowly increasing towards the theoretical result. For $r_{0}=4$, both the power-law exponents of $d$ and $l$ agree reasonably well with the theoretical value $1 / 3$ over the latest times simulated.

A plot of the length-scale ratios $r(t)=d / l$ from the calculations with $r(0)=1$ and 4 is shown in figure 11. As a consequence of the poor resolution of the initial spectrum, the correct value of $r$ is not reproduced from the numerics at $t=0$, and the short-time evolution of $r(t)$ is incorrect. Nevertheless, the long-time evolution of $r(t)$ is in close agreement with the computational results shown in figure 5(a), indicating that the 

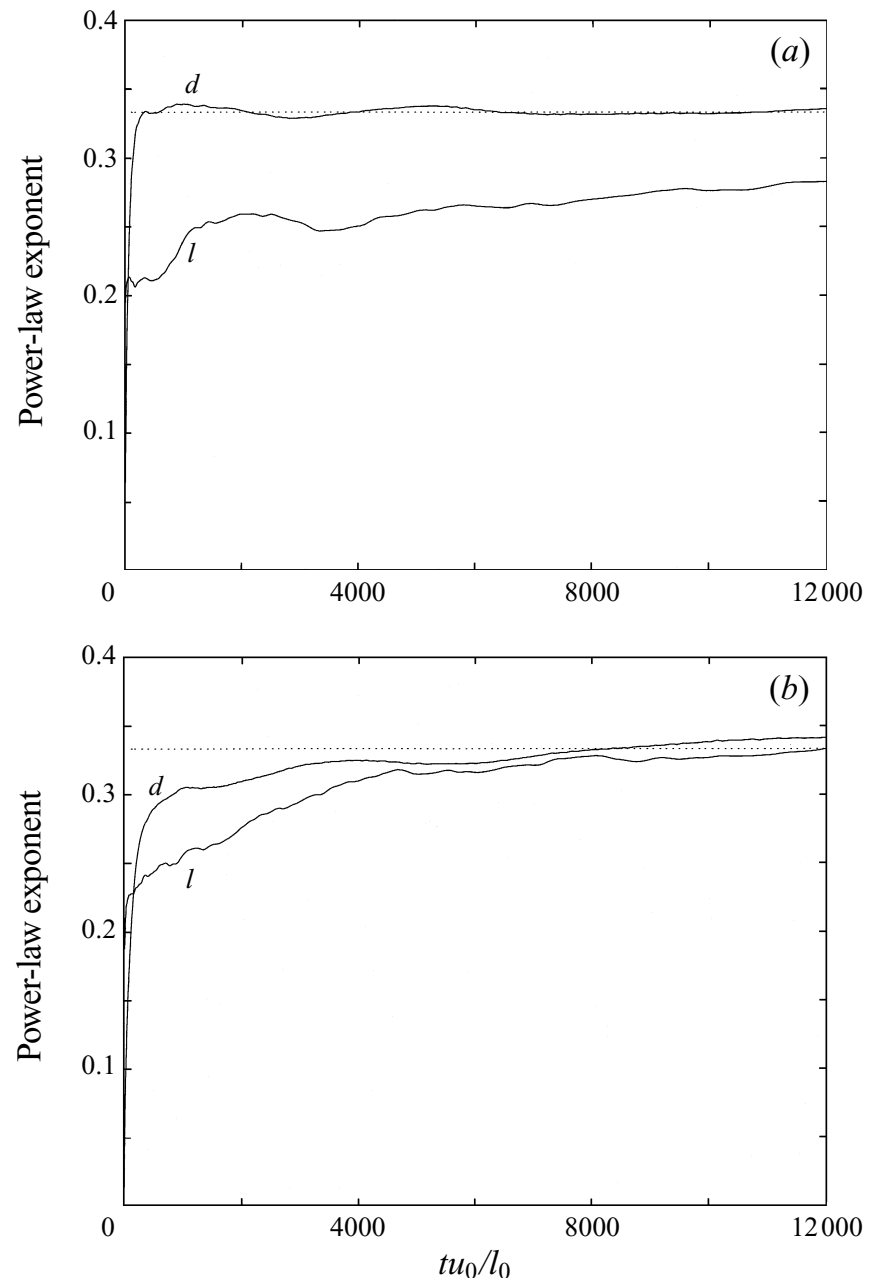

FIGURE 10. Time evolution of the power-law exponents of the length scales $d$ and $l$ for calculations with $k_{p}=126$. (a) $r_{0}=1 ;$ (b) $r_{0}=4$.

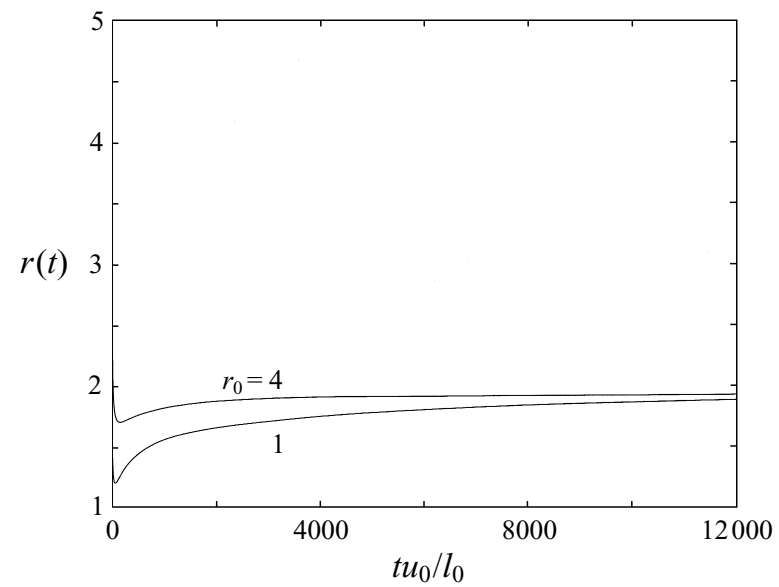

FIGURE 11. Time evolution of the ratio of the length scales $r(t)=d(t) / l(t)$ for initial values $r_{0}=1$ and 4 , and with $k_{p}=126$. 
final similarity state attained in these simulations with $k_{p}=126$ is approximately the same as that attained with $k_{p}=32$.

\section{Conclusions}

The main purpose of this work has been to extend the scaling arguments developed for decaying homogeneous turbulence to the decay of inhomogeneous turbulence in an unbounded domain, thus demonstrating their more general validity. Our analytical and numerical results taken together point to the existence of intermediate asymptotic similarity states at high Reynolds numbers. These similarity states occur for zero to three directions of inhomogeneity - zero directions corresponding to homogeneous turbulence - and depend only on the initial form of the low-wavenumber spectrum associated with the mean-square velocity integrated over the inhomogeneous directions of the flow field.

I would like to thank Professor G. K. Batchelor for his original suggestion that invariant arguments may be applicable to a localized turbulence. I also wish to thank R. S. Rogallo and A. Wray for the use of their simulation software. The computations presented here were performed on an Intel Paragon at The Hong Kong University of Science \& Technology, and the support of the Centre for Computing Services and Telecommunications and the Hong Kong Research Grant Council is gratefully acknowledged.

\section{REFERENCES}

BAtchelor, G. K. 1953 The Theory of Homogeneous Turbulence. Cambridge University Press.

Batchelor, G. K. \& Proudman, I. 1956 The large-scale structure of homogeneous turbulence. Phil. Trans. R. Soc. Lond. 248, 369-405.

Briggs, D. A., Ferziger, J. H., Koseff, J. R. \& Monismith, S. G. 1996 Entrainment in a shear-free turbulent mixing layer. J. Fluid Mech. 310, 215-241.

Chasnov, J. R. 1994 Similarity states of passive scalar transport in isotropic turbulence. Phys. Fluids 6, 1036-1051.

Chollet, J. P. \& Lesieur, M. 1981 Parameterization of small scales of three dimensional isotropic turbulence utilizing spectral closures. J. Atmos. Sci. 38, 2747-2757.

Corral, R. \& Jimenez, J. 1995 Fourier/Chebyshev methods for the incompressible Navier-Stokes equations in infinite domains. J. Comput. Phys. 121, 261-270.

Germano, M., Piomelli, U., Moin, P. \& Cabot, W. H. 1991 A dynamic subgrid-scale eddy viscosity model. Phys. Fluids A 3, 1760-1765.

Gilbert, B. 1980 Diffusion mixing in grid turbulence without mean shear. J. Fluid Mech. 100, $349-365$

Kolmogorov, A. N. 1941 On degeneration of isotropic turbulence in an incompressible viscous liquid. Dokl. Akad. Nauk. SSSR 31538.

Kraichnan, R. H. 1976 Eddy viscosity in two and three dimensions. J. Atmos. Sci. 33, 1521-1536.

Phillips, O. M. 1956 The final period of decay of non-homogeneous turbulence. Proc. Camb. Phil. Soc. 52, 135-151.

Piomelli, U. \& Chasnov, J. R. 1996 Large-eddy simulations: theory and applications. In Turbulence and Transition Modelling (ed. M. Hallback, D. S. Henningson, A. V. Johansson \& P. H. Alfredsson).

Rogallo, R. S. 1981 Numerical experiments in homogeneous turbulence. NASA Tech. Mem. 81315.

SAFFMAN, P. G. 1967a The large-scale structure of homogeneous turbulence. J. Fluid Mech. 27, 581-593.

Saffman, P. G. $1967 b$ Note on decay of homogeneous turbulence. Phys. Fluids 10, 1349.

Veeravalli, S. \& Warhaft, Z. 1989 The shearless turbulence mixing layer. J. Fluid Mech. 207, $191-229$ 